\title{
THE NEXT STEP TOWARDS OPERATIONALIZING RESILIENCE: THE MEASUREMENT OF SELF- SYNCHRONIZATION
}

\author{
Samuel W. Huber ${ }^{1}$ \\ Thomas Kuhn ${ }^{2}$ \\ 1) Forventis, Switzerland \\ 2) Swiss Procurement Agency armasuisse, Switzerland
}

\begin{abstract}
The concept of resilience is becoming widely used by many industries where safety and security is crucial for success. At the last REA we've presented a definition of resilience that can be used in CD\&E experiments regarding socio-technical systems. Self-synchronization was identified to play an important role. Methods: The approach chosen towards diagnosing selfsynchronization was inspired by the methods used in psychiatry to diagnose psychiatric diseases as utilized in the "Diagnostic and Statistical Manual of Mental Disorders" (DSM 5) and the International Classification of Diseases (ICD 10). This approach was chosen because it allows to diagnose certain conditions based solely on the presence or absence of easily observable and assessable conditions and observations. We introduced two categories of indicators. The first category constitutes of five factors (mandate, autonomy, communication, coordination, cooperation) and was named "threshold conditions". These conditions are necessary for self-synchronization to occur but are not sufficient to determine if selfsynchronization occurred. The second category was named "value added observations" and consists of seven factors (situational awareness, self-awareness, negotiation, dependability, motivation, sub-networks, distributed decision making). These observations are used to quantify the level of self-synchronization reached. Results: The model was applied in two different settings of war games, performed during the Multinational Capability Development Campaign (MCDC) "Info-age C2" campaign. It was possible to diagnose the level of selfsynchronization reached in the exercises. Based on these results, the model was further simplified and transferred into a checklist-format that allows for real-time self-synchronization diagnosis during exercises and real-live missions. Conclusion: In this paper, we will present our experience with a newly developed diagnostic model of self-synchronization. A highfidelity research-version will be presented together with an operational version that can be applied for the assessment of self-synchronization during real world missions.
\end{abstract}

Keywords: Self-synchronization, Model, Diagnose, Resilience, Questionnaire, Research, Practice

(C) 2019 Huber, S. W., Kuhn, Th.. This is an Open Access article distributed under the terms of the Creative Commons Attribution-NonCommercial 4.0 International License (http://creativecommons.org/licenses/by-nc/4.0), permitting all noncommercial use, distribution, and reproduction in any medium, provided the original work is properly cited.

ISBN: 978-91-88898-41-8

DOI: https://doi.org/10.15626/rea8.19 


\section{BACKGROUND}

As we have identified in our previous work (Huber \& Kuhn, 2017) self-synchronization seems to be one of the key factors during the degradation phase of an overstrained system. In the view on resilience we have presented in 2017, self-synchronization is one capability that results from the principal of fractality (table 1 and figure 1).

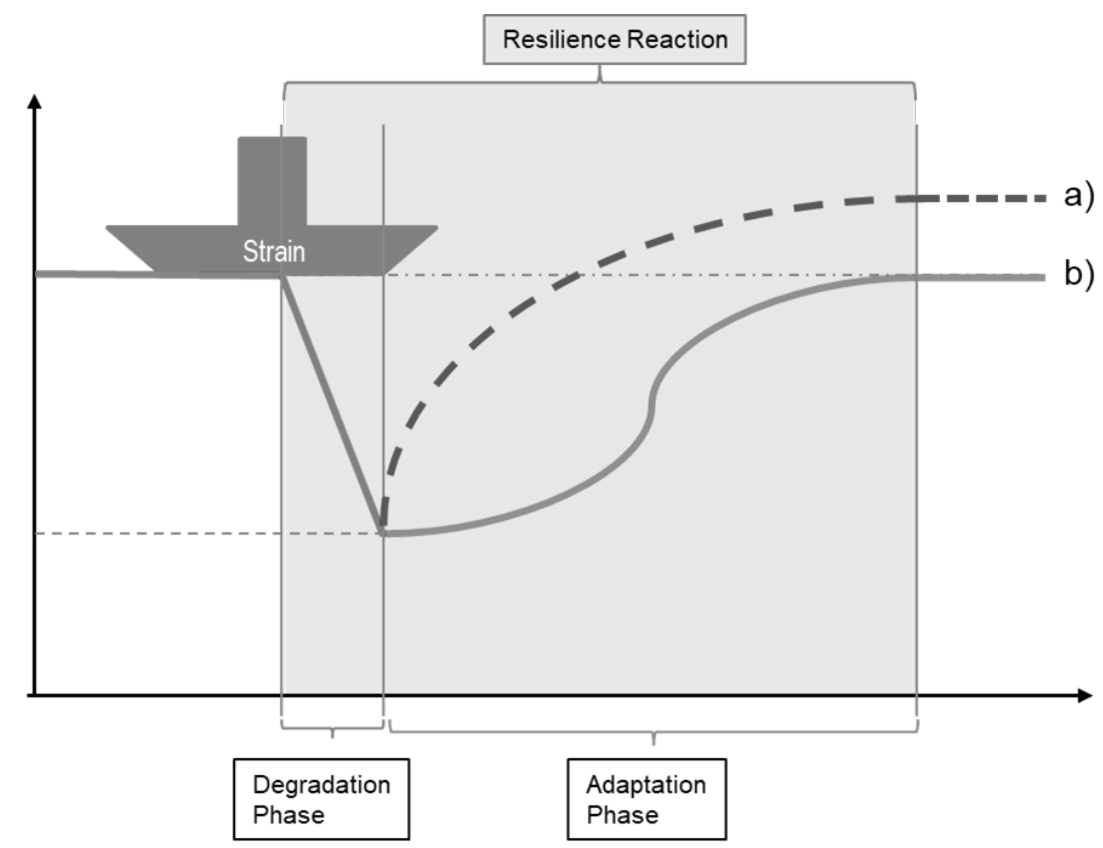

Figure 1. Expression of resilience: phases and typical response curves: a) "Bounce back" b) Adaptation

\begin{tabular}{|llll|}
\hline Phase & Principle & Capability & Mechanism (examples) \\
$\begin{array}{l}\text { Degradation } \\
\text { Phase }\end{array}$ & 1A) Autonomy & - Timely Decision Making & - Involved and Acting \\
& & - Use of Local Intelligence & $\begin{array}{l}\text { Entities Decide for } \\
\text { Themselves }\end{array}$ \\
& 1B) Fractality & - Self-synchronization & - Generic Rules \\
& & - Scalable Organization & - Role-Based \\
& & & Organization \\
& 1C) Compatibility & - Recombinability & - Modularity \\
& & & - Standardization \\
\hline $\begin{array}{l}\text { Adaptation } \\
\text { Phase }\end{array}$ & 2A) Diversity & - Creativity & - T-Shapes \\
& & & (Polyvalence) \\
& & & - Improvisation \\
& 2B) Selection & - Testing & - Trial \& Error \\
& 2C) & - Learning \& & - Adaptive SOP \\
& Standardization & Implementation & \\
\hline
\end{tabular}

Table 1. Element-structure of the resilience framework

In a military setting, Alberts and Hayes (2003) describe self-synchronization as the operating of entities in the absence of traditional hierarchical mechanisms for command and control. 
According to the definition of Cebrowski and Gartska (1998), self-synchronization is the ability of a well-informed force to organize and synchronize complex warfare activities bottom up. Further, Gonzales (2005) sees self-synchronization as the ability of a force to act in a manner, coordinated in intent, time, and space with other battlespace entities, both civilian and military, without being ordered to do so specifically. In other words, the basic idea of selfsynchronization is to push decision making authority down to the entities in the field and thereby replacing the hierarchical approach to leadership. From a resilience point of view, selfsynchronization supports the principle of fractality, which enables organizational stability during the degradation of the system, when teams and units are constantly breaking up and reconfiguring to keep up a functional organization, hence producing a scalable organization. A functional organization is crucial to keep up a significant system output in the state of degradation (Danner-Schroeder \& Geiger, 2016). However, once there are ideas on selfsynchronization implemented, it might be worth testing them in experiments and field studies. In order to do so and to produce objective and valid results, a diagnostic tool is needed which is capable of reliably capturing the relevant aspects of self-synchronization. Therefore, a model has been developed that describes self-synchronization in terms of required and facultative conditions and behaviors. Based on the model, a tool in the format of a questionnaire was developed. There exists a research version that can be used for scientific assessments while the practical version can be used to assess self-synchronization in real-time, without the need for complicated and time-consuming analytical procedures

\section{METHODS}

The approach chosen towards diagnosing self-synchronization was inspired by the methods used in psychiatry to diagnose psychiatric diseases as utilized in the "Diagnostic and Statistical Manual of Mental Disorders" (DSM 5) and the International Classification of Diseases (ICD 10). This approach was chosen because it allows to diagnose certain conditions based solely on the presence or absence of easily observable and assessable conditions and observations (indicators). We use two categories of indicators. The first category was named "threshold conditions". These conditions are necessary and for self-synchronization to appear, all of them must be present. The second category was named "value added observations. These indicators also provide evidence for self-synchronization but are, taken alone, not sufficient to conclude, that self-synchronization occurred. They might still be used to assess the level of selfsynchronization reached.

\subsection{Diagnostic Model for Self-Synchronization (DMS 1.0)}

Based on this methodological approach, in a first attempt four threshold conditions and six value added observations were identified. The indicators were held as abstract as possible to increase the flexibility of use in experimentation.

\section{A) Threshold Conditions}

- Internode Information Exchange. If nodes in the network are not actively exchanging information, then there is no possibility for self-synchronization to occur.

- Interactive/Negotiated Decision Making Observed. There will need to be interactions between nodes to establish prioritization and harmonization of goals, tasks and objectives.

- Adjustments Made (adapting, aligning, deferring). A self-synchronizing network will need to make adjustment to cope with a changing operational environment.

- Intranode (Subnetwork) Self-Assessment. Each node will need to make assessments at each stage in the process model in order to facilitate the NetForce information sharing and decision-making processes. 


\section{B) Value Added Observations}

- Goal setting behaviors observed.

- Flexible Patterns of Interaction observed (deconfliction, coordination, cooperation).

- Stand-alone decisions made by a node based on negotiation.

- Subnetworks established to complete a task or achieve a goal and then ended upon task completion/goal achievement.

- Shared Situational Awareness (Understanding) developed amongst nodes.

- Changes in Operational Environment detected and considered.

The conditions and observations of the model were operationalized and transferred into an online questionnaire set up on LimeSurvey. It then was applied to assess self-synchronization in an experiment with thirteen participants simulating a self-synchronizing network of the same number of nodes.

A) Threshold Conditions

\begin{tabular}{|lr|}
\hline Have you been able to exchange information with other nodes? & $100 \%$ \\
\hline Did you take part in interactive/negotiated Decision Making? & $100 \%$ \\
\hline $\begin{array}{l}\text { Did you make adjustments (adaptations, alignments, deferral) to cope with the changing } \\
\text { operational environment? }\end{array}$ & $92 \%$ \\
\hline Did you perform self-assessments during the game? & $92 \%$ \\
\hline B) Value added observations & $85 \%$ \\
\hline \begin{tabular}{l} 
Did you set goals in cooperation with other nodes? \\
\hline $\begin{array}{l}\text { Have you been involved in flexible patterns of interaction like deconfliction, coordination or } \\
\text { cooperation? }\end{array}$
\end{tabular} & $85 \%$ \\
\hline $\begin{array}{l}\text { Did you made stand-alone decisions based on negotiation? } \\
\text { Have you been part of a temporal subnetwork to complete a task or achieve a goal that ended } \\
\text { after completion/goal achievement? }\end{array}$ & $46 \%$ \\
\hline $\begin{array}{l}\text { Have you been able to build a general (shared) situational awareness (understanding) during the } \\
\text { game? }\end{array}$ & $69 \%$ \\
\hline $\begin{array}{l}\text { Have you been able to detect and consider changes in the "operational environment" during the } \\
\text { game? }\end{array}$ & $62 \%$ \\
\hline
\end{tabular}

Table 2. Assessment of the appearance and quality of self-synchronization. Percent of participants (nodes) in the experiment answering questions with "Yes".

The model was used to assess the presence and level of self-synchronization during the experiment. The results indicated that all the threshold conditions were met while in about $73 \%$ of the nodes, the "value added observations" were reported to be present, indicating that selfsynchronization took place during the experiment though on a medium to high quality level (table 2). However, it was supposed, that the model probably focused too much on the aspect of "synchronization" underweighting the aspect of "self". There were also feedbacks from the participants that the formulations of the questions were hard to be used to assess the situation during the experiment. We've addressed these issues and revised the structure of the model as well as the point of view from which the questions had to be answered by introducing factors 
specific to "self" and "synchronization" and by transforming the generic formulations into descriptions of human behaviors.

\subsection{Revised Diagnostic Model for Self-Synchronization (DMS 2.0)}

In our attempt to replace the generic descriptions of conditions and "value added observations" by better understandable descriptions of human behaviors, we referred to the "Fleishman Job Analysis Survey" (F-JAS; Fleishman et al (1984)), a popular tool for job analysis. Depending on the version, this tool lists a collection of up to 81 human capabilities. We were able to select and use the definitions from nine dimensions of the F-JAS. Three dimensions had to be created specifically for the model.

A) Threshold conditions

The threshold conditions consist of five dimensions that are addressing the aspects of "self" and "synchronization".

a) "Self"

There were two dimensions selected to describe the aspect of "self" in the self-synchronization model. These two factors we've called "mandate" and "autonomy".

- Mandate (new): The mandate provides the guidelines and intended outcome of a NetForce mission. It therefore provides guidance to the nodes on how to prioritize their tasks and actions.

- Autonomy (new): This is the freedom and right of a node to take decisions and prioritize tasks and actions based on its own judgement in favor to achieve the overarching goals.

\section{b) "Synchronization"}

There were three dimensions selected to describe the aspect of "synchronization" in the selfsynchronization model. These three factors we've called "Communication", "Coordination" and "Cooperation".

- Communication (F-JAS): This is the process by which relevant information and intentions are shared by nodes through the exchange of verbal and nonverbal messages.

- Coordination (F-JAS): This is the ability to structure work plans and activities to coincide with others' schedules, style, and pace, and to accommodate necessary changes to schedules. This ability involves effective management of time and materials in order to synchronize actions with other nodes.

- Cooperation (F-JAS): This is the ability of nodes to function effectively in a NetForce - contributing individual abilities towards the attainment of NetForce goals in agreement and coordination with the other nodes. It also involves absence of competition between the nodes.

B) Value added observations

The value-added observations consist of seven dimensions.

- Situational Awareness (F-JAS): This is the state of being always cognizant of the surroundings within a dynamically changing environment. It involves temporal awareness and the anticipation of future events based on the

- Self-Awareness (F-JAS): This is the skill of a node to assess its own performance and capabilities. It involves comparing its own actions and capabilities against task requirements, past outcomes, goals and values.

- Negotiation (F-JAS): This is the process of bargaining with other nodes to resolve conflicts. This involves settling differences through mutual concessions to ensure an acceptable outcome for the nodes involved. 
- Dependability (F-JAS): This is a nodes characteristic of being reliable and responsible to other nodes. This involves being reliable and trustworthy in fulfilling obligations and tasks expected by other nodes in the NetForce.

- Motivation (F-JAS): This is the ability of a node to develop, direct, regulate and maintain effort and energy in order to reach the overarching objective.

- Subnetworks (new): Subnetworks are temporary aggregations of multiple NetForce nodes, established to bundle up capacities and different capabilities in order to complete a task or achieve a goal.

Distributed Decision Making (F-JAS): This is the ability to collectively choose appropriate responses to situations where several options are possible. It involves evaluating several sources of information, option finding and risk assessment.

\section{RESULTS}

\subsection{Questionnaire DMS 2.0r (research version)}

Based on the definitions of the dimensions, we were able to deduct a comprehensive and precise questionnaire that enables the user to assess the level of self-synchronisation and to gather insight into specific aspects of the self-synchronisation. In the research version, 5-point Likert scales were used to allow for analysis on the ordinal level. The items of the questionnaire were derived by asking for an overall assessment of a dimension with the first question (top-level question). We generated further items by extracting every element in the definition and turning it into a question (second-level questions).

The questionnaire is structured as follows:

1. Definition of the dimension

2. Top-level question

3. Second-level questions

\section{A) Threshold Conditions}

- Mandate: The mandate provides the guidelines and intended outcome of a NetForce mission. It therefore provides guidance to the nodes on how to prioritize their tasks and actions.

- Have you been aware of the guidelines and the intended outcome of the NetForce mission?

- How clear were the guidelines and the intended outcome of the NetForce mission to you?

- How useful was the knowledge of the guidelines and the intended outcome of the NetForce mission for the prioritization of tasks and actions?

- Autonomy: This is the freedom and right of a node to take decisions and prioritize tasks and actions based on its own judgement in favour to achieve the overarching goals.

- Have you been able to freely take decisions and prioritize tasks and actions based on your own judgement, in favour to achieve the overarching goals?

- To what degree have you been able to freely take decisions based on your own judgement?

- To what degree have you been able to freely prioritize tasks and actions based on your own judgement?

- Communication: This is the process by which relevant information and intentions are shared by nodes through the exchange of verbal and nonverbal messages.

- Have you been able to share relevant information and intentions with other nodes? 
- How well could you share relevant information and intentions with other nodes?

- Could you share the relevant information and intentions with other nodes in time?

- Coordination: This is the ability to structure work plans and activities to coincide with others' schedules, style, and pace, and to accommodate necessary changes to schedules. This ability involves effective management of time and materials in order to synchronize actions with others.

- Have you been involved in coordination activities?

- How well could you structure work plans and activities to coincide with others' schedules?

- How well could you structure work plans and activities to coincide with others' pace?

- How well could you accommodate necessary changes to schedules?

- How effective could you manage time in order to synchronize actions with others?

- How effective could you manage capabilities in order to synchronize actions with others?

- How effective could you manage capacities in order to synchronize actions with others?

- Cooperation: This is the ability of nodes to function effectively in a NetForce - contributing individual abilities towards the attainment of NetForce goals in agreement and coordination with the other nodes. It also involves absence of competition between the nodes.

- Have you been able to cooperate with other nodes?

- How well could you contribute your individual abilities towards the attainment of NetForce goals?

- How well was the level of agreement you experienced during the cooperation?

- How well was the level of coordination you experienced during the cooperation?

- How effective was the cooperation in order to attain the NetForce goals?

- How much do you agree with the following statement: "There was complete absence of competition between cooperating nodes»?

\section{B) Value Added Observations}

- Situational Awareness: This is the state of being always cognizant of the surroundings within a dynamically changing environment. It involves temporal awareness and the anticipation of future events based on the knowledge of both, the past and the present.

○ Did you experience "Situational Awareness" during the exercise?

- Have you always been cognizant of the surroundings?

- How well was your temporal awareness?

- How well could you anticipate future events based on the knowledge of the past?

- How well could you anticipate future events based on the knowledge of the present?

- Self-Awareness: This is the skill of a node to assess its own performance and capabilities. It involves comparing its own actions and capabilities against task requirements, past outcomes, goals and values. 
○ Did you experience "Self-Awareness" during the exercise?

- How well could you assess your own performance?

- How well could you assess your own capabilities?

- How well could you assess your own capacities?

- How well could you compare your own actions, capabilities and capacities against task requirements?

- How well could you compare your own actions, capabilities and capacities against past outcomes?

- How well could you compare your own actions, capabilities and capacities against goals and values?

- Negotiation: This is the process of bargaining with other nodes to resolve conflicts. This involves settling differences through mutual concessions to ensure an acceptable outcome for the nodes involved.

○ Have you been involved in negotiation activities?

- How effective have you been in bargaining with other nodes to resolve conflicts?

- How effective have you been in settling differences through mutual concessions?

- How successful have you been in achieving an acceptable outcome for the nodes involved?

- Dependability: This is a nodes characteristic of being reliable and responsible to other nodes. This involves being reliable and trustworthy in fulfilling obligations and tasks expected by other nodes in the NetForce.

- Have the nodes you have worked with been dependable?

- How reliable have you been in fulfilling the expectations of other nodes in the NetForce?

- How reliable were the other nodes in fulfilling the obligations and tasks you expected?

- How much trust did you have in the other nodes?

- Motivation: This is the ability of nodes to develop, direct, regulate and maintain effort and energy in order to reach the overarching objective.

- Have you been motivated to reach the overarching objective?

- How much effort and energy have you been able to develop to reach the overarching objective?

- Have you been able to maintain the effort and energy to reach the overarching objective?

- Subnetworks: Subnetworks are temporary aggregations of multiple NetForce nodes, established to bundle up capacities and different capabilities in order to complete a task or achieve a goal.

○ Have you been part of a temporary subnetwork to complete a task or to achieve a goal?

- How well could you complete a task as part of a temporal subnetwork?

- How well could you achieve a goal as part of a temporal subnetwork?

- Could the tasks/goals in the temporary subnetwork be completed/achieved in time?

- How convenient/easy was the process of completing tasks or achieving goals as part of a subnetwork? 
- Distributed Decision-Making: This is the ability to collectively choose appropriate responses to situations where several options are possible. It involves evaluating several sources of information, option finding and risk assessment.

- Have you been involved in distributed decision-making activities?

- How often could you contribute to collective decision making?

- How successful was the process of collectively taking decisions?

- How deeply have you been involved in the evaluation of sources of information?

- How deeply have you been involved in finding options?

- How deeply have you been involved in risk assessment activities?

The items of the research version of the diagnostic model on self-synchronization DMS 2.0r were transferred into a questionnaire. This questionnaire was used to assess the presence and the level of self-synchronization during an experiment performed within a simulation environment during the MCDC project. Aim of the experiment was to get insight in the dynamics of self-synchronization and to gain knowledge on processes that influence the quality of self-synchronization (Information Age Command and Control, 2019). The experiment was supported by a simulation environment in Steel Beast. For communication between the nodes and to provide information to the nodes that cannot be simulated in Steel Beast (e.g. media), Slack was be used. The networked coalition is revered to as NetForce. The participants represented different 'nodes' (= capabilities) in a fictional scenario. Within the scenario all nodes had (individual) objectives, which they should try to meet. The scenario was event driven and the nodes had to respond to the changing situation in the scenario in order to contribute to their objective. While doing so, they encountered other nodes, with whom they could align, or needed to align in order to be able to contribute their objective. The experiment followed a $2 \times 2$ design varying on the conditions 1) provision of communication guidelines, where nodes were either or not provided with an instruction to communicate intended activities and intents .and 2) provision of network information, where an information profile of all nodes that participated in the NetForce was distributed or not resulting in four runs. Ten participants participated each run. We applied DMS 2.0 to look for effects on the appearance of self-synchronization. We were able to capture differences in self-synchronization between different experimental conditions (table 3). Hence, the DMS 2.0 seems to have enough discriminative potential to capture different levels of self-synchronization during the experiment. Further, there were no more critical feedbacks about problems of comprehension or the applicability of the questionnaire to assess the experiment.

\begin{tabular}{|lcccc|}
\hline Results Simulation Experiment (DMS 2.0r) & \multicolumn{3}{l|}{} \\
\hline Experimental Conditions / Run Nr. & 1 & 2 & 3 & 4 \\
\hline Communication Guidelines & No & Yes & No & Yes \\
\hline Network Information & No & No & Yes & Yes \\
\hline Threshold conditions & & & & \\
\hline Mandate & 5 & 3 & 5 & 3 \\
\hline Autonomy & 5 & 5 & 5 & 5 \\
\hline Communication & 5 & 5 & 4.5 & 5 \\
\hline Coordination & 4 & 4.5 & 3 & 4 \\
\hline Cooperation & 5 & 5 & 4.5 & 4 \\
\hline Value Added Observations & & & & \\
\hline Situational Awareness & 4 & 4 & 3 & 3.5 \\
\hline
\end{tabular}




\begin{tabular}{|lcccc|}
\hline Self-Awareness & 4.5 & 4 & 3.5 & 4.5 \\
\hline Dependability & 5 & 4 & 4 & 3 \\
\hline Negotiation & 2 & 1.5 & 1 & 1 \\
\hline Motivation & 6 & 5 & 5 & 5 \\
\hline Temporary Subnetworks & 5 & 5 & 5 & 3.5 \\
\hline Distributed Decision Making & 4 & 5 & 4.5 & 3 \\
\hline
\end{tabular}

Table 3. Results from an experiment in a simulation environment (Steal Beast) with four experimental conditions (communication guidelines / network information accessible). Scores are medians from a 6-point Likert scale ( $1=$ no self-synchronization; $6=$ perfect selfsynchronization). Results for the top-level questions. $N=10$ for each run.

\subsection{Questionnaire DMS 2.0p (practice version)}

The praxis version of the DMS 2.0 differs from the research version in so far, that it only uses the top-level questions. Further, while the research version utilized a 5-point Likert Scale, the praxis version uses a dichotomous scale (yes/no questions). This makes it easier to use the questionnaire in the field, because it allows for an immediate assessment of selfsynchronization reducing the requirement for data processing.

Self-synchronization assessment

For a valid assessment, we suggest that a minimum of four questionnaires should be filed and the following rules applied.

1. To be fulfilled, a condition/factor must be observed (answered with "yes") in $80 \%$ of the cases or more.

2. For self-synchronization to occur, all the five threshold conditions must be observed.

3. Quality assessment for the "Value Added Observations"
a. Very low
$0-19 \%$ "Value Added Observations" observed
b. Low
20-39\% "Value Added Observations" observed
c. Medium 40-59\% "Value Added Observations" observed
d. High 60-79\% "Value Added Observations" observed
e. Very high - 80-100\% "Value Added Observations" observed

\section{DISCUSSION}

The diagnostic model DMS 2.0, as it is, may be a good starting point when it comes to assessing self-synchronization. However, the model must be seen as experimental and is far from being validated. It is well possible that there exist additional dimensions that should be integrated into the model in the future. In so far, the model developed here is still work in progress and should be applied with caution when using it to assess self-synchronization in experiments and field trials to come.

\section{Acknowledgements}

The work presented in this manuscript is based on the result from a team effort from within the MCDC Project "Info-Age C2" (Multinational Capability Development Campaign). The work was further supported by the Swiss Procurement Agency armasuisse. 


\section{REFERENCES}

[1] Alberts, D., Hayes, R. (2019). Power to the Edge: Command...Control...in the Information Age. CCRP Publications.

[2] Cebrowski, A. K., Garstka, J. J. (1998). Network-Centric Warfare: Its Origin and Future. US Naval Institute Proceedings.

[3] Danner-Schroeder, A. \& Geiger, D. (2016). Organisationale Resilienz: Wie Unternehmen Krisen erfolgreich bewältigen können. Zeitschrift Führung und Organisation (pp. 201-2018). Stuttgart: Schäfer-Poeschel Verlag für Wirtschaft, Steuern, Recht GmbH.

[4] Fleishman, E. A., Quaintance, M. K., \& Broedling, L. A. (1984). Taxonomies of human performance: The description of human tasks. San Diego, CA, US: Academic Press.

[5] Gonzales, D. et al. (2015). Network-centric Operations Case Study: The Stryker Brigade Combat Team. RAND National Defense Research Institute.

[6] Huber, S. W., Kuhn, Th (2017). Towards an Operationalizable Definition of Resilience. 7th Resilience Engineering International Symposium Liège (Luik) (Belgium), 26 - 29 June 2017.

[7] Information Age Command and Control: Self-Synchronization (2019). Final Report. Multinational Capability Development Campaign. 